OPEN

SUBJECT AREAS:

NANOPHOTONICS AND

PLASMONICS

METAMATERIALS

Received

29 September 2014

Accepted

21 November 2014

Published

11 December 2014

Correspondence and requests for materials should be addressed to

T.C. (caotun1806@

dlut.edu.cn)

\section{Extrinsic 2D chirality: giant circular conversion dichroism from a metal-dielectric-metal square array}

\author{
Tun Cao, Chenwei Wei, Libang Mao \& Yang Li
}

Department of Biomedical Engineering, Dalian University of Technology, China (P.R.C.)

Giant chiroptical responses routinely occur in three dimensional chiral metamaterials (MMs), but their resonance elements with complex subwavelength chiral shapes are challenging to fabricate in the optical region. Here, we propose a new paradigm for obtaining strong circular conversion dichroism (CCD) based on extrinsic $2 \mathrm{D}$ chirality in multilayer achiral MMs, showing that giant chiroptical response can be alternatively attained without complex structures. Our structure consists of an array of thin Au squares separated from a continuous Au film by a GaAs dielectric layer, where the Au squares occupy the sites of a rectangular lattice. This structure gives rise to a pronounced extrinsically $2 \mathrm{D}$-chiral effect (CCD) in the mid-infrared (M-IR) region under an oblique incidence, where the $2 \mathrm{D}$-chiral effect is due to the mutual orientation of the Au squares array and the incident light propagation direction; the large magnitude of CCD due to the large difference between left-to-left and right-to-right circularly polarized reflectance conversion efficiencies.

( hirality is an intrinsic feature of chiral molecules, which cannot be superposed on their mirror image called enantiometers ${ }^{1}$. This is an ubiquitous property in the organic world, found in proteins, DNA, sugar molecules, viruses, amino acids and cholesteric liquid crystals ${ }^{2}$. Consequently, the analysis of intrinsically 3D-chiral effects has been widely used in the fields of spectroscopy of chemical and biomolecular substances ${ }^{3}$, compact thin-film optical diodes ${ }^{4}$ and circular polarized emission ${ }^{5}$. Meanwhile, $2 \mathrm{D}$ intrinsic chirality is also found in natural planar chiral systems such as the surface of Au monocrystal and the surface of a metal-metal eutectic ${ }^{6}$. However, 2D- and 3D- chiroptical responses in natural chiral materials are usually very weak ${ }^{7}$. They can only be observed once the material exhibits a macroscopic path length of light. This limitation has been lifted by recent advances in both chiral metamaterials $(\mathrm{MMs})^{7-10}$ and non-chiral planar $\mathrm{MMs}^{11-15}$, which have opened new routes towards significant circular dichroism ${ }^{11}$ and optical activity ${ }^{12}-3 \mathrm{D}$ chirality as well as pronounced circular conversion dichroism ${ }^{16}$ and asymmetric transmission-2D chirality ${ }^{17-22}$, surpassing those of natural chiral materials.

Chiral MMs, such as helical structures ${ }^{23-24}$, dual-layer twisted-arc $\mathrm{MMs}^{2}$, twisted U shape split ring $\mathrm{MMs}^{25}$, single-layered gold sawtooth gratings ${ }^{26}$ and multilayer gammadion $\mathrm{MMs}^{27}$ are likely to possess strong chiroptical response. However, periodic arrays with ultrasmall chiral figures and precise alignment are complicated to achieve in the optical region ${ }^{28}$. Therefore, a more challenging task is to achieve chiral effects using intrinsically achiral MMs. This can be obtained by tilting the non-chiral MMs out of their symmetry axes with respect to the incident light ${ }^{11}$. This response is termed extrinsic chirality owing to the non-chiral nature of the structures ${ }^{12}$. Such effects are later demonstrated in various kinds of planar achiral MMs under oblique incident light, such as periodically repeating metallic spheres ${ }^{29}$, asymmetrical split rings $^{30}$, nanorings ${ }^{31}$ or circular nanoholes ${ }^{32}$. Compared with chiral MMs, planar MMs consisting of nonchiral resonators have simpler patterns and are thus easier to realize ${ }^{33}$. Recently, some remarkable research demonstrates that the extrinsic chirality from the planar MMs consisting of non-chiral meta-molecules may lead to strong optical activity ${ }^{11}$, circular dichroism (CD) ${ }^{12}$ and circular conversion dichroism $(\mathrm{CCD})^{1}$. It shows that planar achiral MMs can be used to achieve pronounced extrinsically 3D- and 2D-chiral effects that are identical to those produced by 3D- and 2D-chiral systems. Following these significant works, very recently the concept of extrinsic chirality has been put forward, showing that, achiral MMs based on coupling in a multilayer structure also have a potential to provide a strong chiroptical response. This is because the strong magnetic response caused by the antisymmetric oscillation modes of the two coupled layers can enhance circular polarization difference and circular polarization conversion effects. Therefore, multilayer nonchiral MMs have appealing properties and have drawn much interest. For example, 
Zhang et al. numerically demonstrate a giant circular polarization conversion using layer-by-layer nonchiral $\mathrm{MMs}^{34}$, but this multilayer structure only operates in the $\mathrm{GHz}$ region. Zhao et al. show that a giant and broadband circular polarization can be achieved in multilayer twisted arrays of metasurfaces ${ }^{28}$, whereas the efficiency of the circular polarization selectivity in such structures is strongly dependent on the number of layers, namely the more layers of the structure, the better chiroptical response; this structure with many layers may complicate the fabrication scheme. Thus, an effective method for obtaining the nonchiral MMs with much simpler geometry as well as stronger chiroptical effect in the optical spectral region is desirable and necessary for practical applications.

It has been shown that metamaterial perfect absorbers (MPAs) consisting of two metallic layers spaced apart by a dielectric spacer have extraordinary large absorbance resonances across much of the electromagnetic spectra ${ }^{35-38}$. This phenomenon is attributed to the ability to engineer materials with specified permittivity and permeability responses, allowing for matching the impedance of the MPAs to that of free space hence leading to perfect absorbance ${ }^{39}$. Drawing on insights from this interesting phenomenon, we wondered whether it would be possible to use MPAs for realizing giant extrinsically $2 \mathrm{D}$-chiral effect in the optical regime under off-normal incidence. Our intuitive picture was that a large chiral effect should occur if structures support strong electric and magnetic resonances. It is because the chirality is characterized by electric and magnetic dipolar moments and enhancing chirality spectroscopy entails manipulation of both electric and magnetic fields of light ${ }^{40-42}$. With this idea in mind, we propose an array of thin gold ( $\mathrm{Au}$ ) squares separated from a continuous $\mathrm{Au}$ film by a GaAs dielectric layer, where the Au squares occupy the sites of a rectangular lattice. A rectangular periodic square array is a $2 \mathrm{D}$ anisotropic system that possesses different polarization modes along the two orthogonal lattice vectors. Such an achiral MM that interacts with an oblique incident light can obtain an extrinsically $2 \mathrm{D}$ chirality if it is tilted

(a)

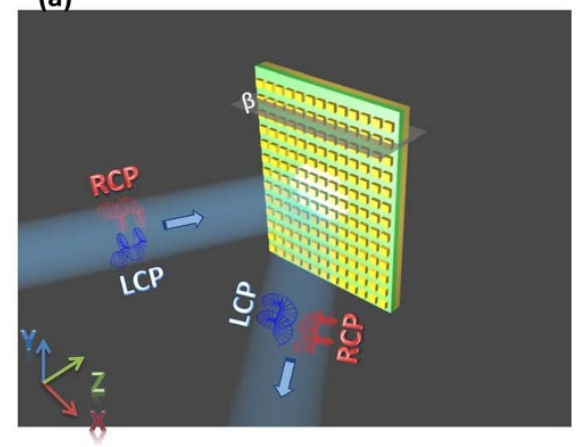

(c)

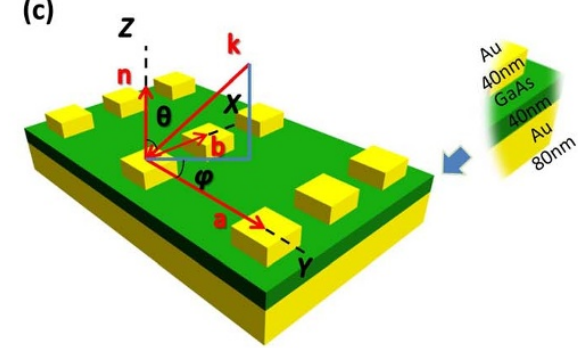

around any in-plane axis that does not coincide with one of the array's lines of mirror symmetry of the MM pattern.

In this work a CCD effect has been investigated to characterize the extrinsic 2D chirality of the structure. Under oblique incidence, our structure gives rise to a giant CCD response in the mid- infrared (MIR) region if only the two principle axes of the structure are not contained in the plane of incidence. The large magnitude of CCD is due to the large difference between the left-to-left and right-toright circularly polarized reflectance conversion efficiencies. Our goal is to show that, by introducing a rectangular geometry in the lattice of MPAs based on metal-dielectric-metal (MDM) multilayers, one can obtain a giant 2D-chiral effect analogous to 2D-chiral MMs in the optical region, while having much simpler patterns that are easier to implement. Moreover, this achiral structure is ultrathin that may be integrated within today's nanophotonic systems. It may be useful for chiral sensing as well as possible applications in circular polarization transformers and waveplates.

\section{Results}

Metamaterials design and simulation method. Figure 1(a) shows the schematic image of the proposed nonchiral MMs, which consists of two gold layers spaced by a $40 \mathrm{~nm}$ thick GaAs dielectric interlayer. The physical origin of high absorbance is the localization of magnetic and electric resonance moments in the metal-dielectric-metal structure. The top Au layer is a $40 \mathrm{~nm}$ thick square gold nanodisk array, where $L_{x}=400 \mathrm{~nm}$ denotes the short side of the rectangular lattice, $L_{y}=800 \mathrm{~nm}$ presents the long side of the rectangular lattice and the side length of the Au square is $d=200 \mathrm{~nm}$. The bottom gold layer has a thickness of $80 \mathrm{~nm}$, which is larger than the penetration depth of light in the optical regime. It will prevent incident light transmitting through the top two layers thus resulting in a nearly zero transmittance. Moreover, by pairing upper squares with a metal ground layer, strong electric and magnetic dipolar resonances are created to couple the incident light into the structure ${ }^{43}$. Therefore, it (b)

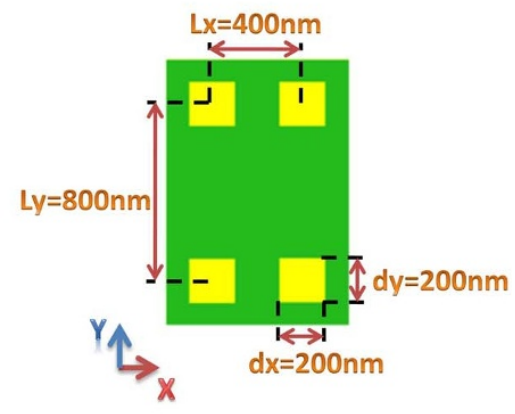

Figure $1 \mid$ (a) Schematic of the MDM-MPAs. A plane wave is incident on the surface of the Au square array. The thicknesses of the Au squares, GaAs spacer, and Au mirror are 40, 40, and $80 \mathrm{~nm}$, respectively. The whole structure is suspended in air. $\beta$ is a cross-section through the structure along the $x-z$ plane. (b) Illustration of absorber's rectangular lattice pattern, where the lattice constant in $x$ direction is $L_{x}=400 \mathrm{~nm}$ and in $y$ direction is $L_{y}=800 \mathrm{~nm}$ and the square dimension is $d_{x}=d_{y}=200 \mathrm{~nm}$. (c) Demonstration of the wavevector $(k)$, the vector normal to the surface ( $n$ ), the two primitive lattice vectors ( $a$ or $b$ ) and the rotation angle $\varphi$ in $x-y$ plane, the components are marked in red. 
can be predicted that the difference in the absorbance (reflectance) for right-handed circularly polarized (RCP) and left-handed circularly polarized (LCP) incident waves may be large under offnormal incidence. The top view of the unit cell is shown in Fig. 1(b), while Fig. 1(c) shows the incident wavevector $k$, the vector normal to the surface $n$, and the two primitive lattice vectors ( $a$ and $b$ ) in red. The incident angle $\theta$ is measured between the wavevector $k$ and the vector $n . \varphi$ is the rotation angle between the parallel (to the $x-y$ plane) component of the wavevector and the $x$ axis. The $z$-axis is normal to the MM surface and the $x-y$ plane is parallel to the surface. The structure is suspended in a vacuum and the $\mathrm{Au}$ squares are periodically extended along the $x$ and $y$ axes. Au is selected as the metal due to its stability and low ohmic loss. We use a finite difference time domain (FDTD) simulator from commercial software '3D EM Explorer Studio' to model the structure. The dielectric properties of $\mathrm{Au}$ as given by Johnson \& Christy are used $^{44}$. A plane wave is incident onto the structure at an oblique angle $\theta$, as described in Fig. 1(c). Periodic boundary conditions were used in the $x$ and $y$ directions. The FDTD mesh size was $2 \mathrm{~nm}$ to provide an accurate calculation on the plasmonic effect. In 3D EM Explorer Studio, we can specify the phases for the $E_{p}$ and $E_{s}$ components, where $E_{p}$ is $p$ polarization of the incident $E$-field and $E_{s}$ is $s$ polarization of the incident $E$ - field. The phase difference between $E_{p}$ and $E_{s}$ is $90^{\circ}$ for RCP and $-90^{\circ}$ for LCP incident light. The $E_{p}$ and $E_{s}$ amplitudes are the same for both LCP and RCP.

Large circular conversion dichroism from a metal-dielectric-metal square array. The complex circular transmission and reflection matrices are defined as $\boldsymbol{E}_{i}=\boldsymbol{t}_{i j} \boldsymbol{E}_{j}^{0}$ and $\boldsymbol{E}_{i}=\boldsymbol{r}_{i j} \boldsymbol{E}_{j}^{0}$, where ' $i$ ' and ' $\boldsymbol{j}$ ' are symbols for RCP $(+)$ and LCP (-) components ${ }^{14}$. The intensities of the corresponding transmitted, reflected, and converted components for RCP and LCP incident light are denoted by $\boldsymbol{T}_{i j}=\left|\boldsymbol{t}_{i j}\right|^{2}$ and $\boldsymbol{R}_{i j}=\left|\boldsymbol{r}_{i j}\right|^{2}$. It has been shown that total transmittance and reflectance through the planar MMs, defined as $T_{j}$ $=T_{j j}+T_{i j}$ and $R_{j}=R_{j j}+R_{i j}$ may be different for circularly polarized waves of either opposite handedness or opposite incident directions ${ }^{11-18}$. However, in our proposed MDM-MPAs the bottom Au layer prevents incident light transmitting through the top two layers hence leading to a nearly zero transmittance. Therefore, here we are mainly focusing on the absolute difference in absorbance of RCP and LCP calculated as $\Delta A=A_{+}-A_{-}$where $A_{ \pm}=1-T_{+}-$ $\boldsymbol{R}_{ \pm}$, as well as the efficiencies of conversion in reflectance $(C C D=$ $R_{--}-R_{++}$) for the circular polarized light incident on the upper square array. Figure $2(a)$ shows the absorbance for RCP (red solid line) and LCP (red dashed line) incident waves with $\theta=\varphi=45^{\circ}, L_{x}$ $=400 \mathrm{~nm}$ and $L_{y}=800 \mathrm{~nm}$. Both RCP and LCP curves possess one main absorbance band. In particular, a near perfect absorbance for RCP incident light $\left(A_{+}=0.86\right)$ is achieved around the resonance wavelength of $2370 \mathrm{~nm}$, whereas the highest absorbance deceases to $A_{-}=0.47$ for LCP light. The absorbance peaks stem from the electric and magnetic resonances in the MDM-MPAs, which can in turn contribute to the strong absorbance difference $(\triangle A)$ as large as 0.39 at $2370 \mathrm{~nm}$ shown in Fig. 2(b). Note that, here $\Delta A$ is due to the fact that the $2 \mathrm{D}$ extrinsic chirality arises from the mutual orientation of non-chiral unit cells and the direction of the light propagation ${ }^{1}$. Therefore, the absorbance of the proposed structure is strongly dependent on the state of circular polarization of the incident light.

Although our proposed MDM-MPA exhibits a near zero transmittance, one could alternatively take advantage of the reflectance spectra for the possible applications of the chiroptical response since the difference of the reflectance of the structure $\Delta R=R_{+}-R_{-}$is also large for the different circular polarized incident light, where $R_{+}$ $=R_{++}+R_{-+}$and $R_{-}=R_{--}+R_{+-}$. Here, $R_{++}, R_{--}, R_{-+}, R_{+-}$ correspond to right-to-right, left-to-left, left-to-right and right-toleft polarized reflectance conversion efficiencies. Figure 3(a) shows that the reflectance at $2370 \mathrm{~nm}$ is $R_{+}=0.14$ for the RCP and $R_{-}=$ 0.53 for the LCP incident light. Nevertheless, $\Delta R$ cannot distinguish between extrinsic 3D chirality (circular dichroism) and $2 \mathrm{D}$ chirality (circular conversion dichroism). In order to resolve this problem, we further look at $R_{++}, R_{--}, R_{+-}$and $R_{-+}$. As can be seen in Fig. 3(b), the diagonal elements $\mathrm{R}_{--}$and $\mathrm{R}_{++}$are not identical indicating that our structure exhibits an extrinsically 2D-chiral reflectance effect, where the circular conversion dichroism is expressed by the difference between the magnitudes of the diagonal elements $C C D=R_{--}$ $-R_{++}$shown in Fig. 3(d). Figure 3(c) shows that the off-diagonal elements of the matrix $R_{-+}$and $R_{+-}$are equal showing the absence of an extrinsic $3 \mathrm{D}$ chirality namely a circular dichroism, which is expressed as $C D=R_{-+}-R_{+-}$. However, the MDM-MPA does not show the asymmetric transmission phenomenon due to the effect of zero transmission.

The giant CCD results from the coupling between the MDM multilayers, which gives rise to a strong magnetic response. Such a strong magnetic dipolar moment is connected to an antisymmetric chargeoscillation eigenmode, providing the combined plasmon mode a twist in the propagation direction of the wave to imitate $2 \mathrm{D}$ chirality $^{45}$. To shed more light on the origin of the large 2D-chiroptical response, we then look at the electromagnetic field distribution in the MDM-MPAs induced by the off-normal incident light. The distributions of total electric field intensity $\boldsymbol{E}=\sqrt{\left|\boldsymbol{E}_{x}\right|^{2}+\left|\boldsymbol{E}_{\boldsymbol{y}}\right|^{2}+\left|\boldsymbol{E}_{z}\right|^{2}}$, total magnetic field intensity $\boldsymbol{H}=\sqrt{\left|\boldsymbol{H}_{\boldsymbol{x}}\right|^{2}+\left|\boldsymbol{H}_{\boldsymbol{y}}\right|^{2}+\left|\boldsymbol{H}_{z}\right|^{2}}$ and displacement current $\left(J_{D}\right)$ along $\beta$ plane associated with $A_{+}=0.86$ at $\lambda=$ $2370 \mathrm{~nm}$ for the RCP incident light at $\theta=\varphi=45^{\circ}$ are shown in Fig. 4(a)-4(c). In the field maps of Fig. 4 , the arrows show $J_{D}$ whereas the color shows the magnitude of the electric field and magnetic field.
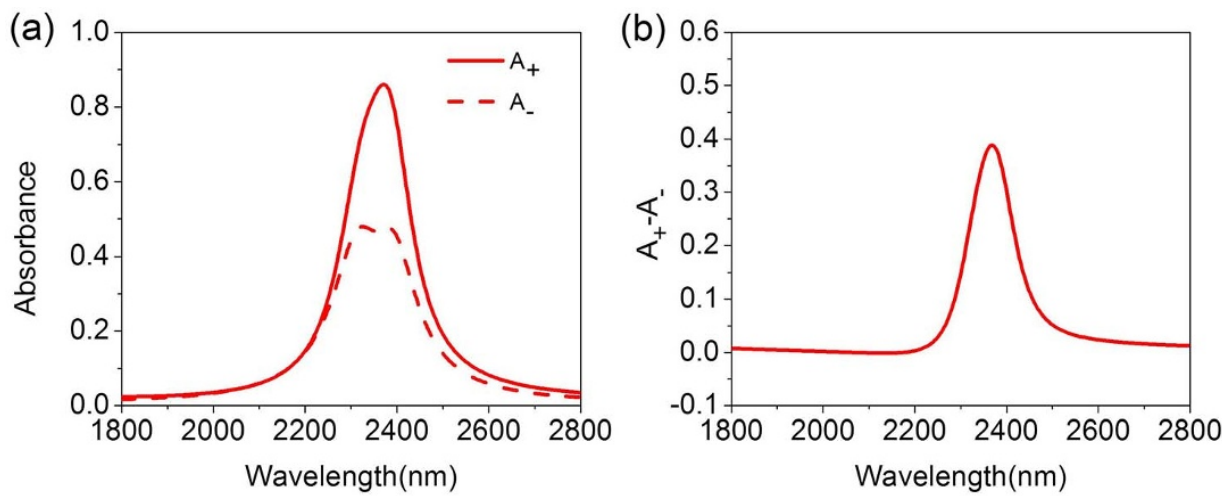

Figure 2 Spectra for right and left circularly polarized light incident at angle $\theta=\varphi=45^{\circ}$ (a) the absorbance of MDM-MPAs with $L_{x}=400 \mathrm{~nm}$ and $L_{Y}$ $=800 \mathrm{~nm} ;(\mathrm{b}) \Delta \mathrm{A}=\mathrm{A}_{+}-\mathrm{A}_{-}$for MDM-MPAs with $L_{x}=400 \mathrm{~nm}$ and $L_{Y}=800 \mathrm{~nm}$ at $\theta=\varphi=45^{\circ}$. 

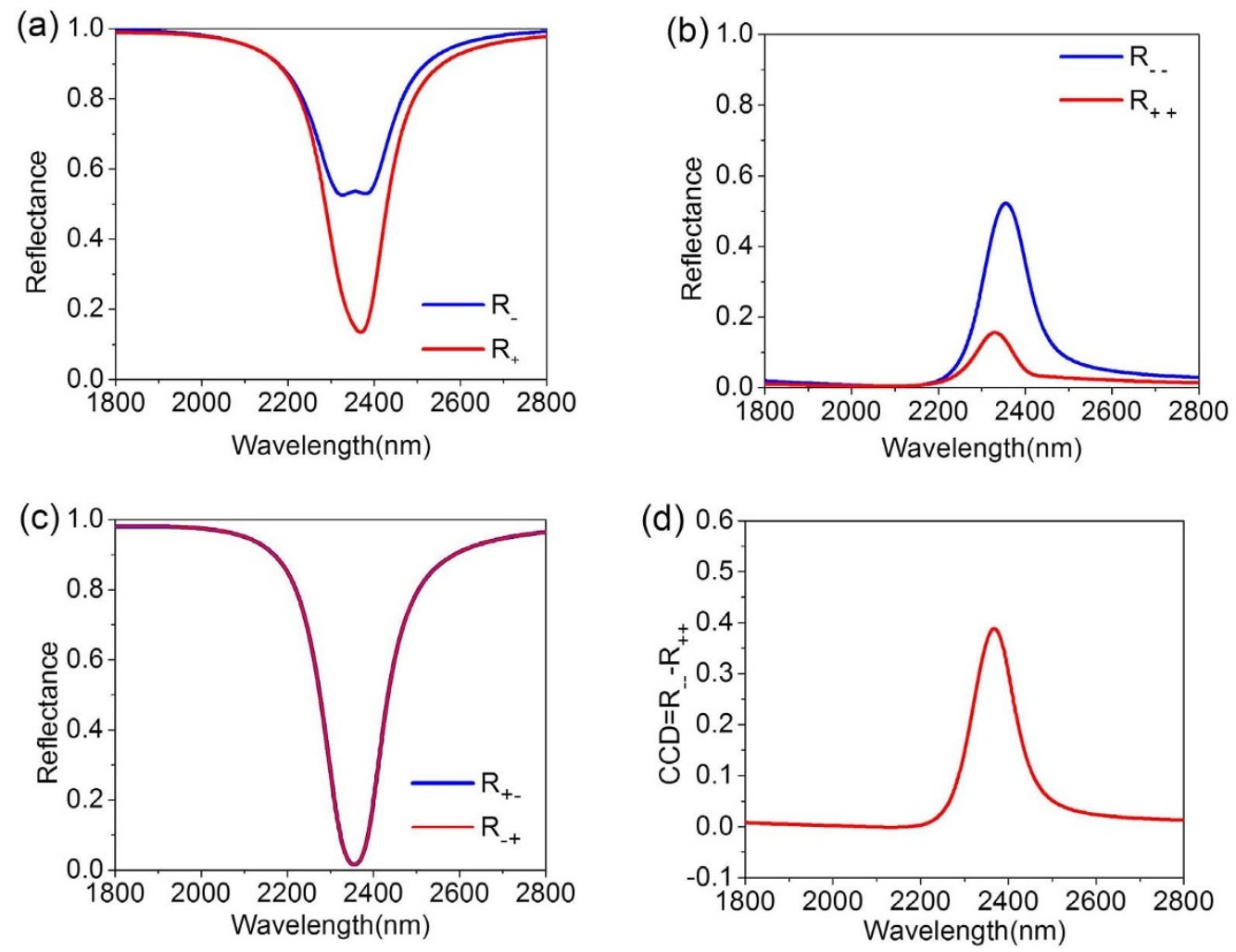

Figure 3 Spectra for right and left circularly polarized light incident at angle $\theta=\varphi=45^{\circ}$ (a) the spectra of $R_{-}$and $R_{+} ;$(b) the spectra of $R_{--}$and $R_{++}$; (c) the spectra of $R_{+-}$and $R_{-+}$; (d) the spectra of $C C D=R_{--}-R_{++}$of MDM-MPAs with $L_{x}=400 \mathrm{~nm}$ and $L_{y}=800 \mathrm{~nm}$.

Figure 4(a) shows that the $E$ field can be localized in both the aperture between the $\mathrm{Au}$ squares and the GaAs dielectric interlayer. Meanwhile, as can be seen in Fig. 4(b) the $H$ field is efficiently confined in the GaAs layer between the Au layers. This is due to the formation of the $J_{D}$ loops shown in Fig. 4(c) and a concomitant coupling between surface plasmons counterpropagating on the two closely spaced interfaces ${ }^{46}$. Therefore, this structure provides a simultaneous excitation of an electric and magnetic resonance moment to enhance the localized electromagnetic fields. Correspondingly, the structure exhibits a near perfect absorbance for the RCP incident light. Figure 4(d)-4(f) show $E, H$ and $J_{D}$ respectively, associated with $A_{-}=0.47$ at $\lambda=2370 \mathrm{~nm}$ for the LCP incident light at $\theta=\varphi=45^{\circ}$. It shows that the patterns of $E$ and $H$ field distribution are similar with the ones for the RCP incident wave, which indicates that the electric and magnetic dipolar resonances are also simultaneously excited to lead to a main peak in the absorbance spectra. However, both $E$ and $H$ field intensities are weaker, which is consistent with the much lower absorbance shown in Fig. 2(a).

The thickness of the dielectric layer is important since it affects the dipole resonance, i.e. the resonance effect is diminished and the absorbance is reduced (reflectance is increased) with increasing thickness ${ }^{43}$. A comparison of CCD response at angle $\theta=\varphi=45^{\circ}$ for the different thicknesses of GaAs dielectric interlayer $T_{d}=$ $40 \mathrm{~nm}, 60 \mathrm{~nm}, 80 \mathrm{~nm}$ and $100 \mathrm{~nm}$ is presented in Fig. 5. It can be seen that the various thicknesses of the dielectric interlayer have a significant effect on the value and frequency band of the CCD curve: the maximum CCD is decreased and the spectra of the CCD is shifted as the thickness of GaAs layer is increased. It is because the frequency of the magnetic resonant dipole in the MDM-MPAs is shifted once we change the dielectric layer thickness, thus the magnetic moment needs to be relocated by means of redesigning the geometry of the $\mathrm{Au}$ squares to obtain the impedance match to a vacuum ${ }^{47}$. As a result, the best performance of the $2 \mathrm{D}$-chiral effect may appear at diverse angles of oblique incident wave for the various $T_{d}$.
To show how the CCD spectra evolve when changing the ratios of the sides of the rectangular lattice $\left(L_{y} / L_{x}\right)$, we show in Fig. 6 the CCD evolution for fixed $L_{x}=400 \mathrm{~nm}$ and $\theta=\varphi=45^{\circ}$ as the values of the $L_{y}$ of the rectangular lattice are varied. It appears that we are unable to obtain a $2 \mathrm{D}$-chiral response $(\mathrm{CCD}=0)$ for a square array of the $\mathrm{Au}$ squares $\left(L_{x}=L_{y}=400 \mathrm{~nm}\right)$ at $\varphi=45^{\circ}$ as the plane of incidence falls on a line of mirror symmetry of the structure. By increasing the ratio of $L_{y} / L_{x}$, the value of CCD is gradually increased. However, for larger ratio values of $L_{y} / L_{x}$, the CCD response is suppressed. This is most likely due to the fact that the coupling of neighboring Au squares along the y direction is reduced by increasing the value of $L_{y}$, since the rectangular lattice becomes dilute.

Figure 7(a) presents the CCD spectra for different values of rotation angle $\varphi$ at a fixed incident angle $\theta=45^{\circ}$. The spectral band and shape of the CCD spectra depend weakly on $\varphi$. The maximum value of CCD increases with $\varphi$, whereas it starts being reduced when $\varphi$ becomes larger than $45^{\circ}$. We note that the circular difference effects vanishes for the structure orientation $\varphi=0^{\circ}$ and $90^{\circ}$, in which case the anisotropic axis of the structure is in the incident plane hence leading to a mirror plane of the experimental geometry ${ }^{48}$. Importantly, the CCD spectra reverse the sign for opposite values of $\varphi$, which corresponds to two enantiomeric arrangements. This will certainly eliminate various problems with the fabrication of exact mirror image left- and right- intrinsically chiral structures. In Fig. 7(b), we also study the $\theta$ effect on the CCD spectra by fixing $\varphi=45^{\circ}$. As expected, there is no chiroptical response at normal incidence $\left(\theta=0^{\circ}\right)$. Particularly, the CCD response increases with $\theta$, it can be significantly enhanced for $\theta>30^{\circ}$ and obtains the maximum value of 0.39 at $2370 \mathrm{~nm}$ for $\theta=45^{\circ}$. Therefore, in our simulation the $2 \mathrm{D}$-chiroptical response is optimized at $\varphi=\theta=45^{\circ}$. Particularly, opposite signs of incidence lead to the same chiroptical effect. Namely, the spectra of CCD shown in Fig. 7(b) do not reverse for opposite angles of incidence ${ }^{13}$, which satisfies the definition of extrinsic 2D chirality. 

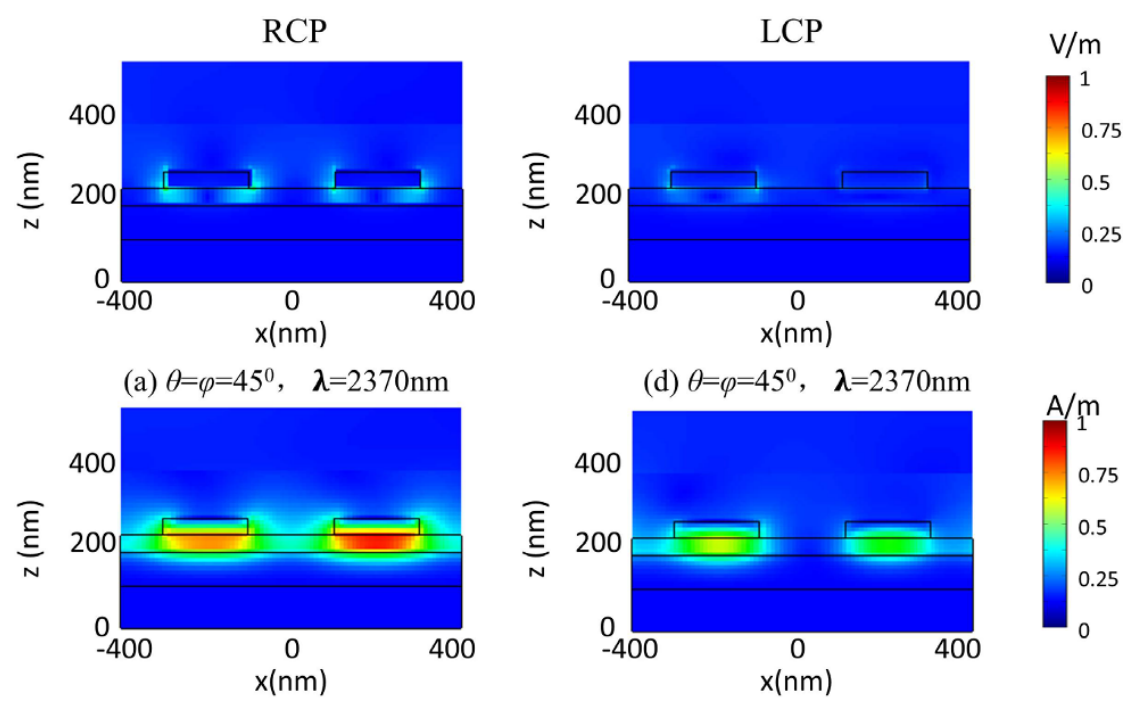

(b) $\theta=\varphi^{=} 45^{0}, \quad \lambda=2370 \mathrm{~nm}$

(e) $\theta=\varphi=45^{0}, \quad \lambda=2370 \mathrm{~nm}$

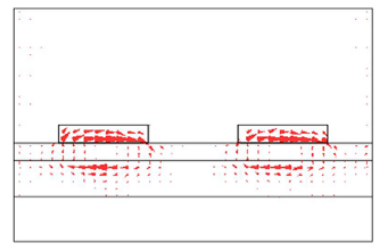

(c) $\theta=\varphi=45^{0}, \quad \lambda=2370 \mathrm{~nm}$

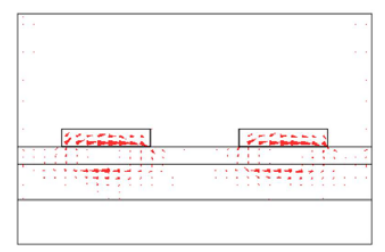

(f) $\theta=\varphi=45^{0}, \quad \lambda=2370 \mathrm{~nm}$

Figure 4 3D-FDTD simulation of (a) total electric field intensity distribution, (b) total magnetic field intensity distribution, and (c) displacement current $\left(J_{D}\right)$ distribution for the RCP incident light at $\theta=\varphi=45^{\circ}$ where $\lambda=2370 \mathrm{~nm}$. Simulation of (d) total electric field intensity distribution, (e) total magnetic field intensity distribution, and (f) displacement current $\left(J_{D}\right)$ distribution for the LCP incident light at $\theta=\varphi=45^{\circ}$ where $\lambda=2370 \mathrm{~nm}$.

In Fig. 8(a), we present two CCD spectra for normal $\left(\theta=\varphi=0^{\circ}\right)$ incidence where $2 \mathrm{D}$ chirality is absent, and for oblique $\left(\theta=\varphi=45^{\circ}\right)$ incidence where a substantial $2 \mathrm{D}$-chiroptical response exists. Figure $8(\mathrm{~b})-8(\mathrm{c})$ show the snapshots of the $E$ field distributions at $\lambda=2370 \mathrm{~nm}$ on the Au square array-air interface with RCP and LCP incident waves under normal incidence $\left(\theta=\varphi=0^{\circ}\right)$, it shows that the field patterns for the two circular polarization are exact mirror images of each other. The difference of these field patterns cancels out hence leading to a zero CCD. Figure $8(\mathrm{~d})-8(\mathrm{e})$ show that on tilting the structure i.e. $\theta=\varphi=45^{\circ}$, the field patterns are very different between the different circularly polarized waves and a clear asymmetry appears in the snapshots, which is due to the fact that a

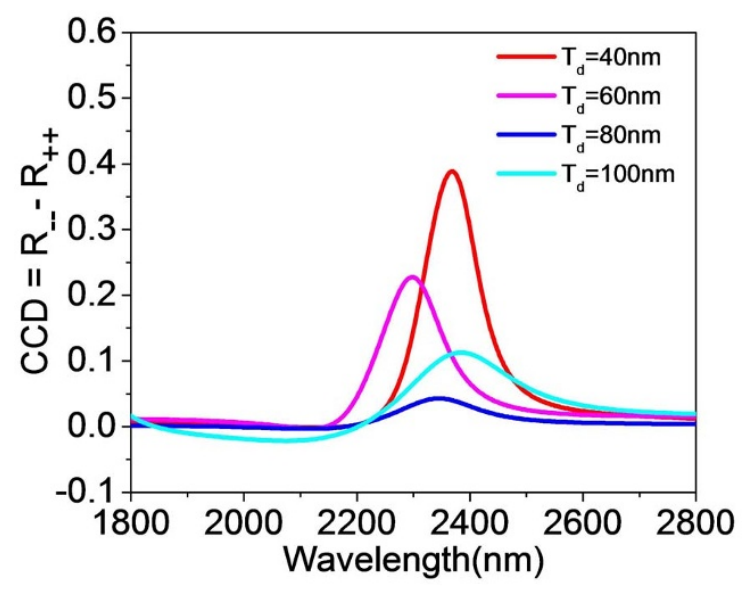

Figure $5 \mid$ Circular conversion dichroism for $\varphi=\theta=45^{\circ}$ with different thicknesses of GaAs dielectric interlayer in MDM-MPAs. various, asymmetric plasmon modes are excited by the RCP and LCP waves. Moreover, the $E$ field is not distributed uniformly over the square array under the off-normal incidence. It is because that the time of the pulse propagating through different regions of the structure is unequal. Different circularly polarized light gives rise to the phase of the various resonance modes that cause constructive or deconstructive interferences of the modes at the squares. This results in a significant difference in the reflectance and absorbance of the structure between the RCP and LCP waves.

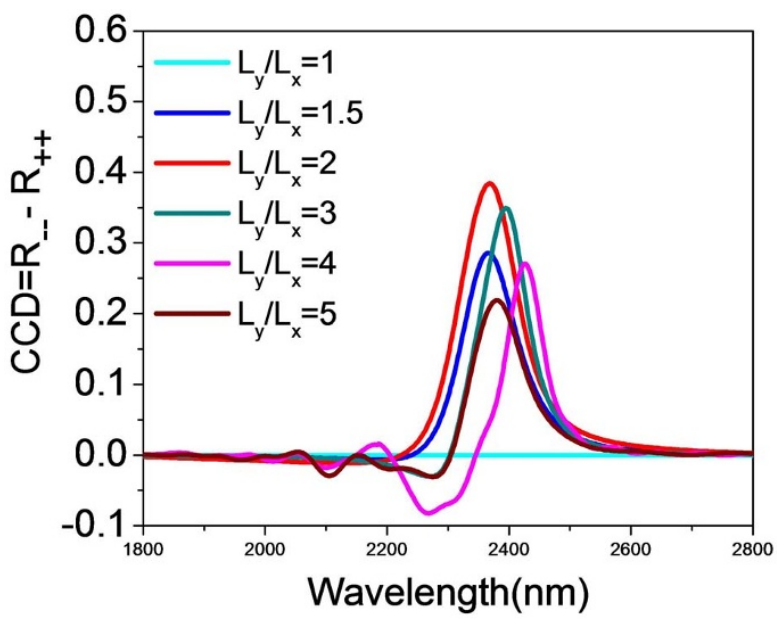

Figure $6 \mid$ Circular conversion dichroism for $\varphi=\theta=45^{\circ}$ with different ratios of $L_{y} / L_{x}$ in the MDM-MPAs. 

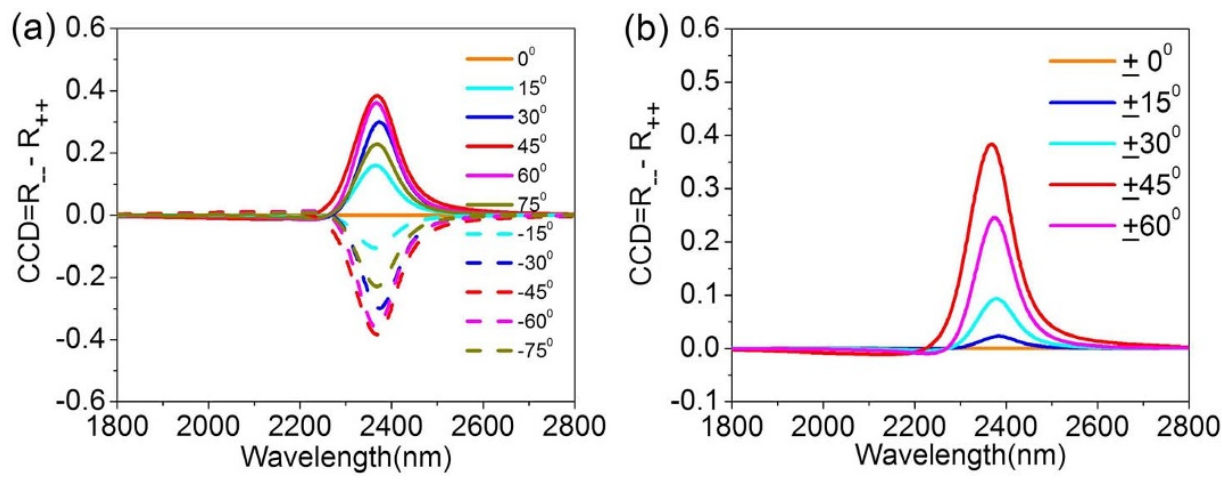

Figure $7 \mid$ Circular conversion dichroism for (a) $\theta=45^{\circ}$ incidence with different values of $\varphi$; (b) $\varphi=45^{\circ}$ incidence with different values of $\theta$.
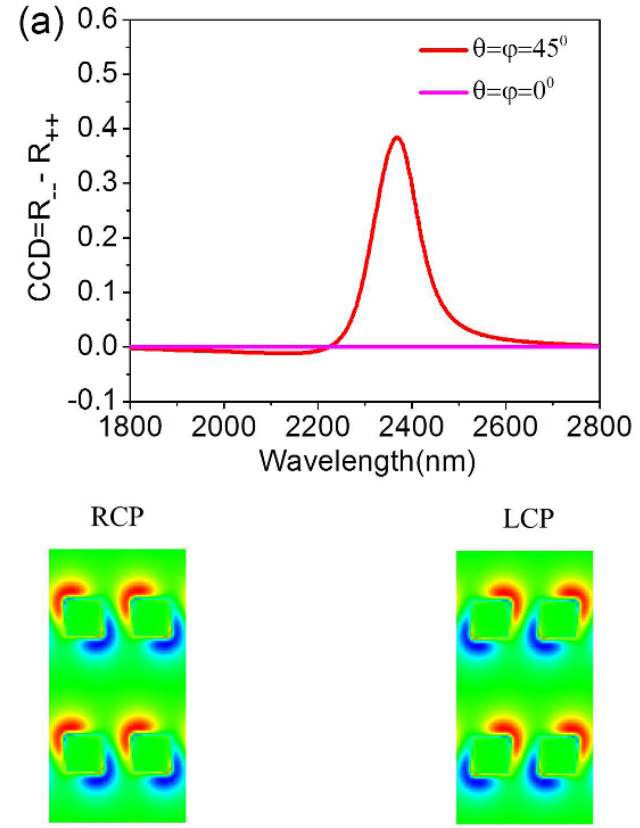

(b) $\theta=\varphi=0^{0}, \quad \lambda=2370 \mathrm{~nm}$

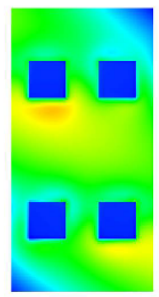

(d) $\theta=\varphi=45^{\circ}, \quad \lambda=2370 \mathrm{~nm}$

Figure 8 (a) FDTD simulation of the circular conversion dichroism spectra of the MDM-MPA at normal $\left(\theta=\varphi=0^{\circ}\right)$ and oblique $(\theta=\varphi=$ $45^{\circ}$ ) incidences. (b) Snapshots of normalized electric field distribution at the Au square array-air during light propagation through the MDM-MPA with $\theta=\varphi=0^{\circ}$ at $\lambda=2370 \mathrm{~nm}$ for RCP incident light. (c) Snapshots of normalized electric field distribution at the Au square array-air during light propagation through the MDM-MPA with $\theta=\varphi=0^{\circ}$ at $\lambda=$ $2370 \mathrm{~nm}$ for LCP incident light. (d) Snapshots of normalized electric field distribution at the Au square array-air during light propagation through the MDM-MPA with $\theta=\varphi=45^{\circ}$ at $\lambda=2370 \mathrm{~nm}$ for RCP incident light. (e) Snapshots of normalized electric field distribution at the Au square array-air during light propagation through the MDM-MPA with $\theta=\varphi=$ $45^{\circ}$ at $\lambda=2370 \mathrm{~nm}$ for LCP incident light.

\section{Discussion}

In conclusion, we have demonstrated that a large extrinsically 2Dchiroptical response in the M-IR region can be observed in highly symmetric plasmonic square arrays based on MDM multilayers, where the Au squares occupy the sites of a rectangular lattice. This extrinsic 2D chirality results from the mutual orientation of the MDM-MPAs and the oblique incident wave. It is thought that the giant CCD is related to the strong magnetic dipole moment. Furthermore, our structure can obtain the two "enantiomers" from the same array by tilting at opposite angles of in-plane rotation $(\varphi)$, which will avoid the problems with the fabrication of exact mirror image left- and right- intrinsically chiral structures. Our structure may be directly integrated into nanophotonic devices owing to its ultrathin profile. Its simple geometry is very amenable to large area advanced nanofabrication techniques and could thus become an important enabling technology in numerous photonics applications. Our proposed concept may be easily scaled down to the shorter wavelength region for a variety of exciting applications in the visible regime.

\section{Methods}

For the numerical calculations, we used the finite difference time domain (FDTD) Method by means of commercial software '3D EM Explorer Studio'. The scheme of the nonchiral MMs is shown in Fig. 1(a), the top view of the unit cell is shown in Fig. 1(b), while Fig. 1(c) shows the incident wavevector $k$, the vector normal to the surface $n$, and the two primitive lattice vectors $(a$ and $b$ ) in red. The incident light is incident onto the structure at an oblique angle $\theta$ in order to achieve the extrinsic $2 \mathrm{D}$ chirality. To account for the periodic nature of the MM, the model boundary at $x= \pm \frac{L_{x}}{2}$ and $y= \pm \frac{L_{y}}{2}$ is set to periodic boundary conditions, respectively. Perfect matched layer is applied in the $\mathrm{z}$ direction. The optical constant of gold is taken from the data of Johnson and Christy ${ }^{41}$. The FDTD mesh size was $2 \mathrm{~nm}$ to provide an accurate calculation on the plasmonic effect. In 3D EM Explorer Studio, we can specify the phases for the $E_{p}$ and $E_{s}$ components, where $E_{p}$ is $p$ polarization of the incident $E$-field and $E_{s}$ is s polarization of the incident $E$ - field. The phase difference between $E_{p}$ and $E_{s}$ is $90^{\circ}$ for RCP and $-90^{\circ}$ for LCP incident light. The $E_{p}$ and $E_{s}$ amplitudes are the same for both LCP and RCP.

1. Plum, E., Fedotov, V. A. \& Zheludev, N. I. Extrinsic electromagnetic chirality in metamaterials. J. Opt. A: Pure Appl. Opt. 11, 074009 (2009).

2. Cui, Y., Kang, L., Lan, S., Rodrigues, S. \& Cai, W. Giant Chiral Optical Response from a Twisted-Arc Metamaterial. Nano Lett. 14, 1021-1025 (2014).

3. Ranjbar, B. \& Gill, P. Circular Dichroism Techniques: Biomolecular and Nanostructural Analyses- A Review. Chem. Biol. Drug Des. 74, 101-120 (2009).

4. Hwang, J. et al. Electro-tunable optical diode based on photonic bandgap liquidcrystal heterojunctions. Nat. Mater. 4, 383-387 (2005).

5. Kopp, V. I., Fan, B., Vithana, H. K. M. \& Genack, A. Z. Low-threshold lasing at the edge of a photonic stop band in cholesteric liquid crystals. Opt. Lett. 23, 1707-1709 (1998).

6. Fullman, R. L. \& Wood, D. L. Origin of spiral eutectic structures L'origine des structures eutectiques, spiraliformes Der ursprung der eutektischen spiralstrukturen. Acta Metall. 2, 188 (1954).

7. Ziolkowski, R. W. \& Engheta, N. [INTRODUCTION, HISTORY, AND SELECTED TOPICS IN FUNDAMENTAL THEORIES OF METAMATERIALS] Metamaterials: Physics and Engineering Explorations 
[Engheta, N. \& Ziolkowski, R. W.(ed.)] [5-37] (John Wiley \& Sons \& IEEE Press, New Jersey, 2006).

8. Liu, Y. \& Zhang, X. Metamaterials: a new frontier of science and technology. Chem. Soc. Rev. 40, 2494-2507 (2011).

9. Plum, E., Fedotov, V. A., Schwanecke, A. S., Zheludev, N. I. \& Chen, Y. Giant optical gyrotropy due to electromagnetic coupling. Appl. Phys. Lett. 90, 223113 (2007).

10. Plum, E. et al. Metamaterial with negative index due to chirality. Phys. Rev. B 79, 035407 (2009).

11. Plum, E. et al. Metamaterials: optical activity without chirality. Phys. Rev. Lett. 102, 113902 (2009).

12. Plum, E., Fedotov, V. A. \& Zheludev, N. I. Optical activity in extrinsically chiral metamaterial. Appl. Phys. Lett. 93, 191911 (2008).

13. Plum, E., Fedotov, V. A. \& Zheludev, N. I. Asymmetric transmission: a generic property of two-dimensional periodic patterns. J. Opt. 13, 024006 (2011).

14. Plum, E., Fedotov, V. A. \& Zheludev, N. I. Planar metamaterial with transmission and reflection that depend on the direction of incidence. Appl. Phys. Lett. 94 131901 (2009).

15. Singh, R., Plum, E., Zhang, W. \& Zheludev, N. I. Highly tunable optical activity in planar achiral terahertz metamaterials. Opt. Express 18, 13425-13430 (2010).

16. Schwanecke, A. S. et al. Nanostructured Metal Film with Asymmetric Optical Transmission. Nano Lett. 8, 2940-2943 (2008).

17. Fedotov, V. A., Schwanecke, A. S. \& Zheludev, N. I. Asymmetric Transmission of Light and Enantiomerically Sensitive Plasmon Resonance in Planar Chiral Nanostructures. Nano Lett. 7, 1996-1999 (2007).

18. Fedotov, V. A. et al. Asymmetric Propagation of Electromagnetic Waves through a Planar Chiral Structure. Phys. Rev. Lett. 97, 167401 (2006).

19. Menzel, C. et al. Asymmetric Transmission of Linearly Polarized Light at Optical Metamaterials. Phys. Rev. Lett. 104, 253902 (2010).

20. Shi, J. H. et al. Dual-band asymmetric transmission of linear polarization in bilayered chiral metamaterial. Appl. Phys. Lett. 102, 191905 (2013).

21. Shi, J. H. et al. Broadband chirality and asymmetric transmission in ultrathin $90^{\circ}-$ twisted Babinet-inverted metasurfaces. Phys. Rev. B 89, 165128 (2014).

22. Xu, Y. Q., Shi, Q. C., Zhu, Z. \& Shi, J. H. Mutual conversion and asymmetric transmission of linearly polarized light in bilayered chiral metamaterial. Opt. Express 22, 25679-25688 (2014).

23. Gansel, J. K. et al. Gold helix photonic metamaterial as broadband circular polarizer. Science 325, 1513-1515 (2009).

24. Christofi, A., Stefanou, N., Gantzounis, G. \& Papanikolaou, N. Giant Optical Activity of Helical Architectures of Plasmonic Nanorods. J. Phys. Chem. C. 116, 16674-16679 (2012).

25. Mutlu, M., Akosman, A. E., Serebryannikov, A. E. \& Ozbay, E. Asymmetric chiral metamaterial circular polarizer based on four U-shaped split ring resonators. Opt. Lett. 36, 1653-1655 (2011).

26. Gao, W. et al. Circular dichroism in single-layered gold sawtooth gratings. J. Opt. Soc. Am. B. 29, 3021-3026 (2012).

27. Cao, T. et al. Strongly tunable circular dichroism in gammadion chiral phasechange metamaterials. Opt. Express. 21, 27841-27851 (2013).

28. Zhao, Y., Belkin, M. A. \& Alù, A. Twisted optical metamaterials for planarized ultrathin broadband circular polarizers. Nat. Commun. 3, 870 (2012).

29. Yannopapas, V. Circular dichroism in planar nonchiral plasmonic metamaterials. Opt. Lett. 34, 632-634 (2009).

30. Shi, J. H. et al. Tunable symmetric and asymmetric resonances in an asymmetrical split-ring metamatrial. J. Appl. Phys. 112, 073522 (2012).

31. Feng, C., Wang, Z. B., Lee, S., Jiao, J. \& Li, L. Giant circular dichroism in extrinsic chiral metamaterials excited by off-normal incident laser beams. Opt. Commun. 285, 2750-2754 (2012)

32. Maoz, B. M., Moshe, A. B., Vestler, D., Bar-Elli, O. \& Markovich, G. Chiroptical Effects in Planar Achiral Plasmonic Oriented Nanohole Arrays. Nano Lett. 12, 2357-2361 (2012).

33. Volkov, S. N. et al. Optical activity in diffraction from a planar array of achiral nanoparticles. Phys. Rev. A. 79, 043819 (2009).

34. Zhang, P. et al. Giant circular polarization conversion in layer-by-layer nonchiral metamaterial. J. Opt. Soc. Am. A. 30, 1714-1718 (2013).
35. Landy, N. I., Sajuyigbe, S., Mock, J. J., Smith, D. R. \& Padilla, W. J. Perfect Metamaterial Absorber. Phys. Rev. Lett. 100, 207402 (2008).

36. Feng, Q., Pu, M., Hu, C. \& Luo, X. Engineering the dispersion of metamaterial surface for broadband infrared absorption. Opt. Lett. 37, 2133-2135 (2012).

37. Wang, J. et al. Tunable broad-band perfect absorber by exciting of multiple plasmon resonances at optical frequency. Opt. Express. 20, 14871-14878 (2012).

38. Fedotov, V. A., Mladyonov, P. L., Prosvirnin, S. L. \& Zheludev, N. I. Planar electromagnetic metamaterial with a fish scale structure. Phys. Rev. E 72, 056613 (2005).

39. Shrekenhamer, D., Chen, W. \& Padilla, W. J. Liquid Crystal Tunable Metamaterial Absorber. Phys. Rev. Lett. 110, 177403 (2013).

40. García-Etxarri, A. \& Dionne, J. A. Surface-enhanced circular dichroism spectroscopy mediated by nonchiral nanoantennas. Phys. Rev. B 87, 235409 (2013).

41. Tang, Y. \& Cohen, A. E. Optical Chirality and Its Interaction with Matter. Phys. Rev. Lett. 104, 163901 (2010).

42. Tang, Y. \& Cohen, A. E. Enhanced Enantioselectivity in Excitation of Chiral Molecules by Superchiral Light. Science 332, 333-336 (2011).

43. Zhang, B. et al. Polarization-independent dual-band infrared perfect absorber based on a metal-dielectric-metal elliptical nanodisk array. Opt.Express. 19 15221-15228 (2011).

44. Johnson, P. B. \& Christy, R. W. Optical constants of the noble metals. Phys. Rev. B. 6, 4370-4379 (1972).

45. Decker, M., Klein, M. W., Wegener, M. \& Linden, S. Circular dichroism of planar chiral magnetic metamaterials. Opt. Lett. 32, 856-858 (2007).

46. Dayal, G. \& Ramakrishna, S. A. Design of highly absorbing metamaterials for Infrared frequencies. Opt. Express 20, 17503-17508 (2012).

47. García-Meca, C. et al. Double-negative polarization independent fishnet metamaterial in the visible spectrum. Opt. Lett. 34, 1603-1605 (2009).

48. Verbiest, T., Kauranen, M., Persoons, A. \& Persoons, A. Optical activity of anisotropic achiral surfaces. Phys. Rev. Lett. 77, 1456-1459 (1996).

\section{Acknowledgments}

We acknowledge the financial support from National Natural Science Foundation of China (Grant No. 61172059 and 51302026), Ph.D Programs Foundation of Ministry of Education of China (Grant No. 20110041120015), Postdoctoral Gathering Project of Liaoning Province (Grant No. 2011921008), and The Fundamental Research for the Central University (Grant No. DUT14YQ109).

\section{Author contributions}

T.C. conceived the idea of using plasmonic nanorods array based on MDM multilayers, where the Au squares occupy the sites of a rectangular lattice, for obtaining a large extrinsically $2 \mathrm{D}$-chiral response in the M-IR region. T.C. designed the metamaterial and performed numerical analysis. C.W.W. and L.B.M. carried out the simulations, Y.L. prepared the figures. T.C. supervised the research and wrote the manuscript.

\section{Additional information}

Competing financial interests: The authors declare no competing financial interests. How to cite this article: Cao, T., Wei, C., Mao, L. \& Li, Y. Extrinsic 2D chirality: giant circular conversion dichroism from a metal-dielectric-metal square array. Sci. Rep. 4, 7442; DOI:10.1038/srep07442 (2014).

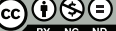

This work is licensed under a Creative Commons Attribution-NonCommercialNoDerivs 4.0 International License. The images or other third party material in this article are included in the article's Creative Commons license, unless indicated otherwise in the credit line; if the material is not included under the Creative Commons license, users will need to obtain permission from the license holder in order to reproduce the material. To view a copy of this license, visit http:/ creativecommons.org/licenses/by-nc-nd/4.0/ 